\title{
Removal of Pentachlorophenol from Water Using Novel Smart Hydrogel Microspheres
}

\author{
Krzysztof Szczubiałka, Anna Karewicz, Łukasz Moczek, Karolina Zomerska, \\ Maria Nowakowska*
}

Faculty of Chemistry, Jagiellonian University, Ingardena 3, 30-060 Kraków, Poland. Fax: +48 12 6340515. Email: nowakows@chemia.uj.edu.pl

(Received: 10 May, 2006; published: 16 June, 2006)

\begin{abstract}
The thermoresponsive hydrogel microspheres were obtained by copolymerization of $\mathrm{N}$-isopropylacrylamide (NIPAM) and 2-hydroxyethyl methacrylate (HEMA) crosslinked with $N, N^{\prime}$-methylenebisacrylamide (BIS). The hydrogel was then functionalized using 1-naphthyl glycidyl ether (NGE) to attach naphthyl chromophores, which absorb UV light and act as photosensitizers. The ability of the functionalized microspheres to photosensitize dechlorination reactions was confirmed using pentachlorophenol (PCP) as a model compound.
\end{abstract}

\section{Introduction}

The smart (also called intelligent or adaptive) materials are getting ever-increasing interest both because of their unusual properties and because of the growing number of their possible practical applications [1-6]. Among them an important group of materials are smart or stimuli-responsive polymers. They can be defined as polymers, whose properties may change drastically in response to the application or a small change in some stimulus, e.g. temperature [7, 8], $\mathrm{pH}[9,10]$, electric field [11, 12], and magnetic field [13, 14]. The possible applications of smart polymers include shape memory materials [15], intracellular delivery of therapeutic macromolecules [16], gene delivery [17, 18], microfluidic devices [19], and polymer-biomolecule conjugates [20] to mention just a few and the list is still rapidly growing.

In a series of our previous papers we have shown that water-soluble amphiphilic polymeric photosensitizers containing rose bengal [21], carbazole [22-24], anthracene [25, 26], naphthalene [27], or porphyrin [28], can be used in the photodegradation of the compounds of environmental interest. We have also devised a smart water-soluble polymer based on NIPAM containing rose bengal chromophores which can be used in the photosensitized oxidation of phenol in the aqueous media [29]. The present paper is an extension of these studies to smart hydrogel polymers. We have obtained smart hydrogel composed of NIPAM and HEMA in the form of microspheres, which were then functionalized with naphthyl chromophores and studied as potential photosensitizers in the reactions of environmental interest such as dechlorination of chlorinated aromatic pollutants (CAPs). CAPs are generally highly toxic compounds. They show cancerogenic, teratogenic and mutagenic activity. Unfortunately, because of their numerous applications they are widespread in the environment, especially in the aquatic environments, where they tend to bioaccumulate in the living organisms. Therefore, there is a need for the development of efficient methods of CAPs detoxification, which do not show the drawbacks of conventional methods like incineration or 
chemical degradation. This paper provides an alternative method of water purification based on a smart hydrogel which can photosensitize degradation of a chlorinated pollutant present in water.

\section{Results and Discussion}

\section{Synthesis and Properties of Microspheres}

The primary goal of the present study was to obtain a smart polymeric material which could be used to carry out photosensitized reactions of environmental interest in the aqueous media. The optimal polymer for such an application should be soluble or at least swellable in water. In the second case the highly porous polymeric microspheres should be preferable. They have a large surface exposed to water which should facilitate the diffusion-controlled contact of the pollutant molecules dissolved in water with the active sites in polymer. Such thermosensitive microspheres were obtained by $\mathrm{Ni}$ et al [30]. They are composed of HEMA and NIPAM crosslinked with BIS. Their swelling ratios decrease monotonically with increasing temperature by a factor of $2.5-4$ in the temperature range of $20-50^{\circ} \mathrm{C}$, the response to temperature being more noticeable for microspheres with higher content of NIPAM [30]. What is also important from the point of view of the goal of our study is that the microspheres were obtained using HEMA which form biodegradable physical hydrogels and whose hydroxyl groups can be easily derivatized. Therefore, we have applied their procedure to obtain microspheres composed of NIPAM and HEMA copolymerized at 9:1 molar ratio using BIS as the crosslinker. The chemical structure of the microspheres is given in Scheme 1.

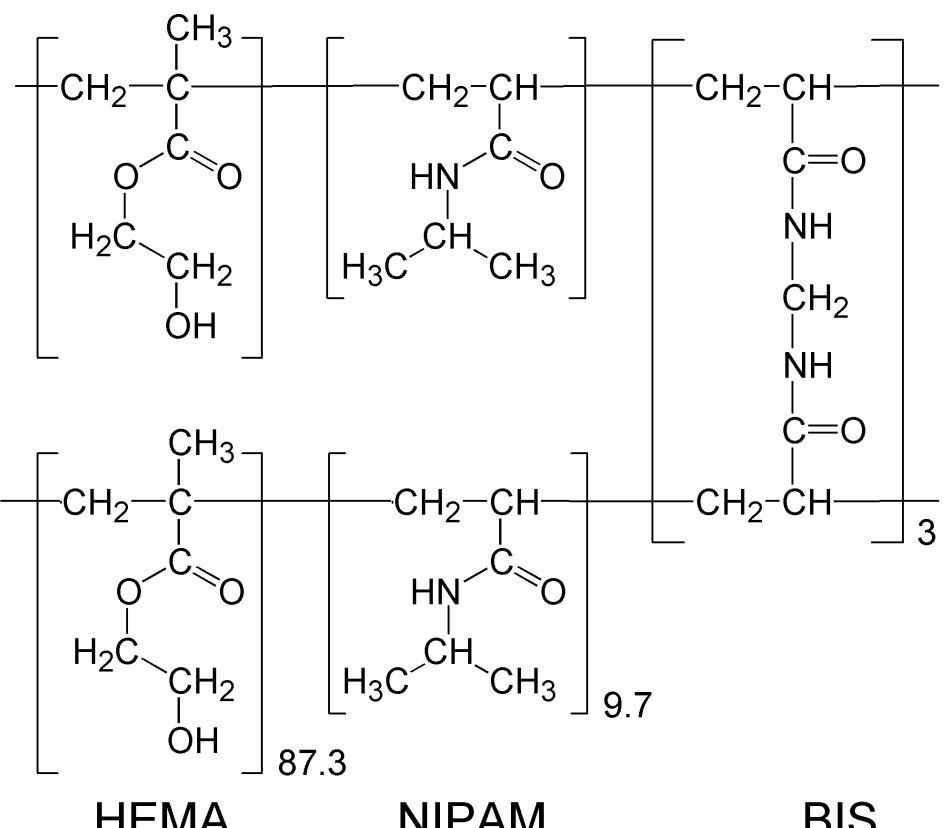

Scheme 1. The chemical structure of the microspheres.

In order to make photoactive microspheres the HEMA hydroxyl groups were then substituted with NGE to attach naphthyl chromophores (Scheme 2). 


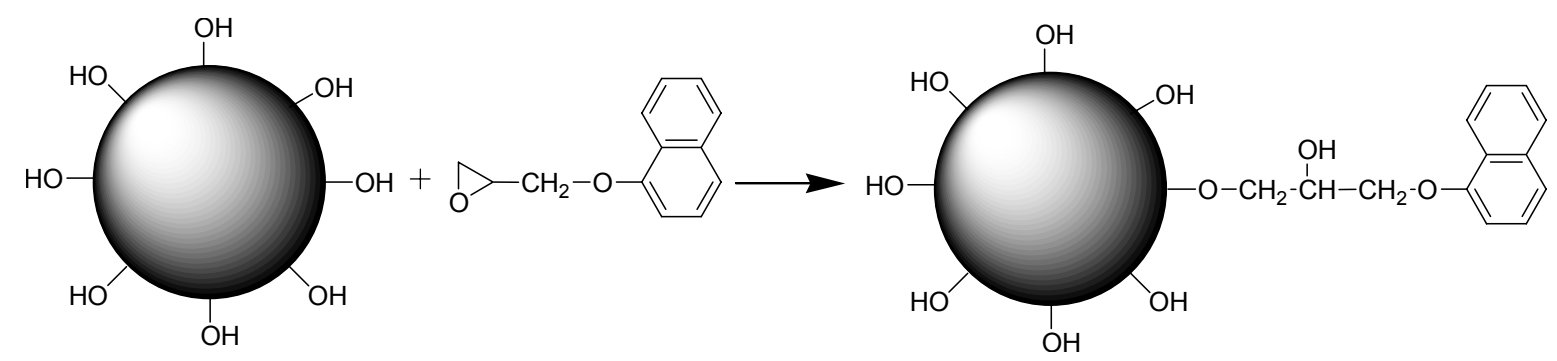

Scheme 2. Derivatization of microspheres with naphthyl chromophores.

We have obtained the microspheres with two different degrees of substitution of HEMA hydroxyl groups with NGE, i.e. $18.5 \%$ and $89.0 \%$, as found from elemental analysis. The microspheres obtained were assigned acronyms NHNp1 and NHNp2, respectively (labeled with naphthyl (Np) chromophores).

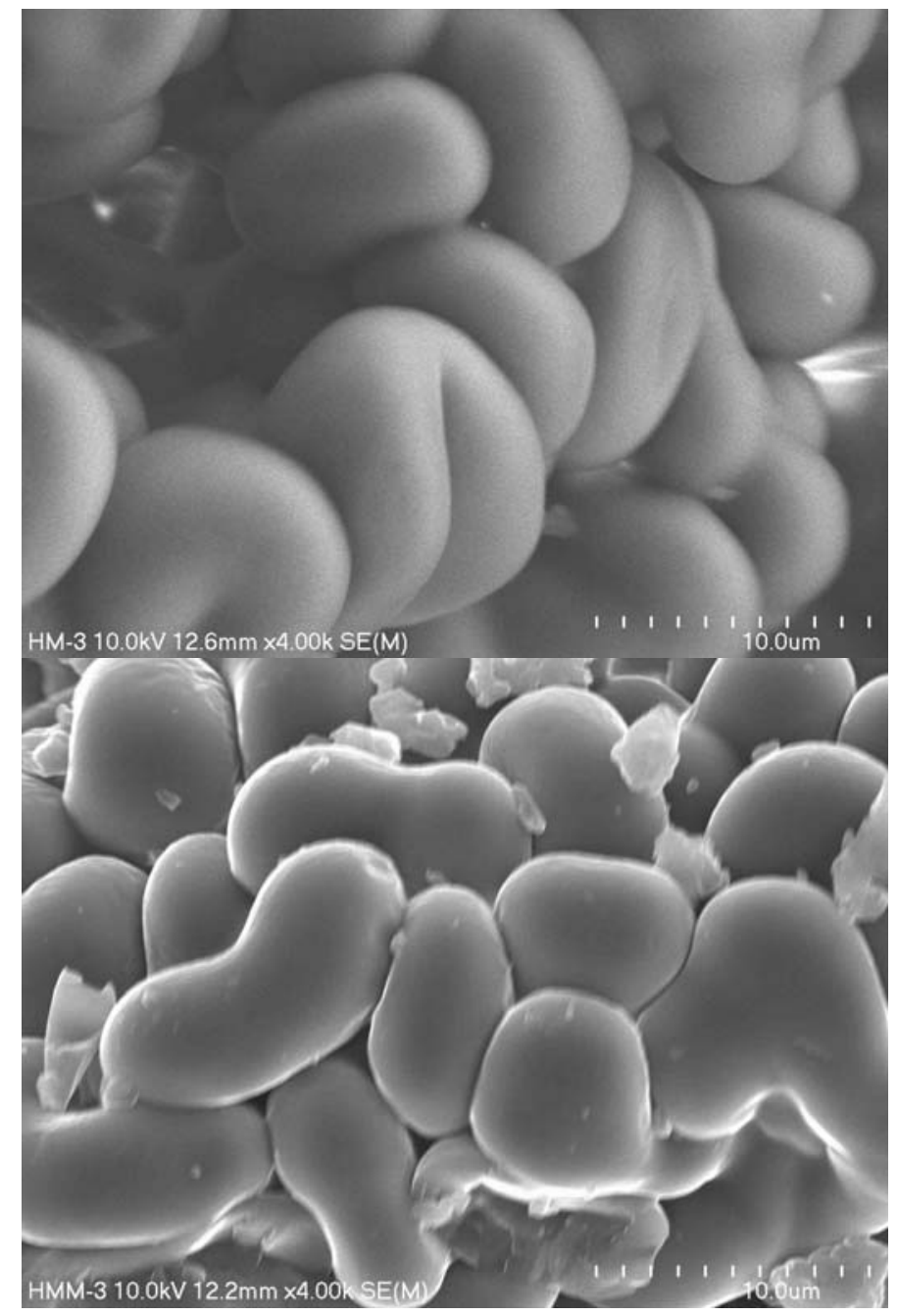

Fig. 1. SEM photographs of microspheres before (upper photo) and after derivatization with NGE (lower photo).

The substitution with NGE was confirmed with UV absorption and fluorescence emission spectra showing the naphthalene moiety absorption band at about $280 \mathrm{~nm}$ and emission band at approximately $340 \mathrm{~nm}$ (see, for example, Figure 2b). Taking 
into account the fact that the microspheres are highly porous one may assume that they are homogeneously substituted with NGE throughout their whole volume. Therefore, the area of contact between the photochemically active hydrogel phase and the bulk solution is large and efficient photosensitized dechlorination is expected.

Detailed information on the morphology of the microspheres was achieved using SEM microscopy of the freeze-dried polymer (Figure 1). Apparently, the sample preparation caused significant distortion of the shape of the microspheres which became oval rather than spherical. Sample preparation could also be the reason why some part of the polymer was found to be amorphous. The SEM photographs have confirmed that some of the microspheres were intimately associated with each other in pairs or even triplets during the polymerization process forming larger structures. It also seems that the derivatization of the microspheres with NGE resulted in the decrease of their average size.

\section{Photosensitized by microspheres degradation of PCP in aqueous solution}

To study the possibility of the application of the photoactive, smart hydrogel microspheres as a photosensitizer of the dechlorination of CAPs we have selected pentachlorophenol (PCP) as a model compound because of its high chlorine content and substantial solubility in water. The solutions of PCP in 15 vol.- $\%$ of methanol in water (small fraction of methanol was added to increase the solubility of PCP) were irradiated in Rayonet photoreactor (lamps $350 \mathrm{~nm}$ ) with in the presence of the microspheres. The solutions for irradiations were prepared by adding different amounts of microspheres to the same stock solution of PCP $\left(c=6.5 \cdot 10^{-5} \mathrm{~mol} \cdot \mathrm{dm}^{-3}\right)$. The emission band of the lamps used was wide enough so that the light could be absorbed by the naphthyl chromophores (absorption band at about $280 \mathrm{~nm}$ ) (Figure 2a). However, as can bee seen in Figure 2a PCP also absorbs that radiation (absorption band with maximum at $303 \mathrm{~nm}$ ) so PCP was expected to undergo direct photodechlorination. Therefore, we have performed the irradiations of PCP also in the absence of the microspheres to find out if the addition of the microspheres results in faster dechlorination of PCP due to photosensitization by naphthyl chromophores. The fluorescence emission spectra of NHNp1 microspheres (Figure 2b) provide more evidence of successful functionalization of the microspheres with Np chromophores. Moreover, the fluorescence spectrum of the microspheres in water shows a shoulder at the long wavelength side of the fluorescence band compared to the corresponding spectrum in methanol. This is due to the formation of excimers by Np chromophores in water and indicates that the chromophores move comparatively freely that they can assume mutual parallel conformation necessary to form excimers. The freedom of motion of the naphthyl chromophores is desirable because it increases the probability of the direct contact of excited naphthyl chromophore and PCP molecules during the excited state lifetime of the former. Formation of the excimers, however, may decrease the quantum yield of the energy transfer from $\mathrm{Np}$ chromophores to PCP molecule by providing another route of the deactivation of the excited state of $\mathrm{Np}$. 

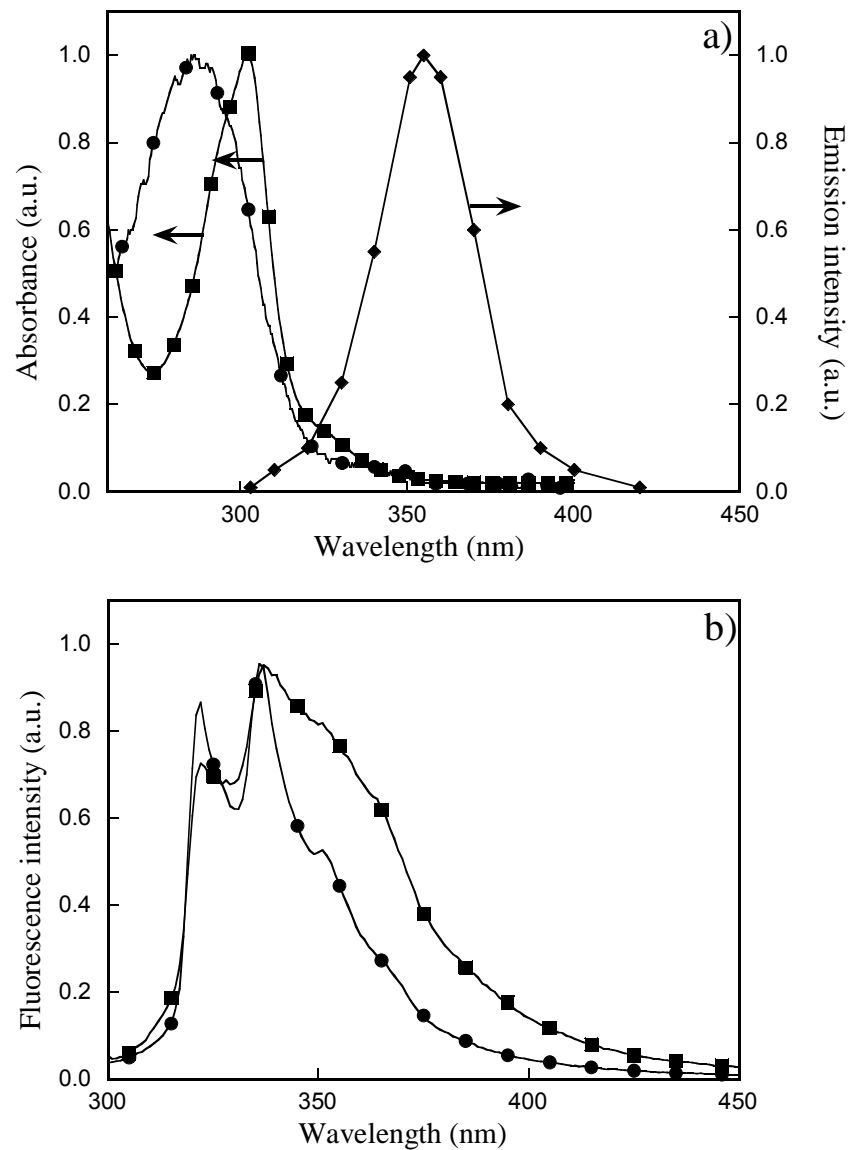

Fig. 2. a) Normalized electronic absorption spectra of NHNp1 microspheres ( $c=1$ $\left.\mathrm{g} \cdot \mathrm{dm}^{-3}\right)(\mathbf{O})$ and PCP $\left(\mathrm{c}=6.5 \cdot 10^{-5} \mathrm{~mol} \cdot \mathrm{dm}^{-3}\right)(\boldsymbol{\square})$ in water and emission band of lamps used for irradiation $(\diamond)$; b) Normalized fluorescence emission spectra of the NHNp1 microspheres $\left(\lambda_{\mathrm{ex}}=280 \mathrm{~nm}, 1 \mathrm{~g} \cdot \mathrm{dm}^{-3}\right)$ in methanol $(\mathbf{O})$ and in water $(\mathbf{\square})$.

The changes in PCP concentration with irradiation time were traced with HPLC after removing the microspheres by filtration. Figure 3 shows the results for the irradiations of PCP solution in the absence and in the presence of NHNp1 microspheres.

The data in Figure 3 show that the irradiation of the PCP results in the decrease of its concentration with irradiation time both in the absence and in the presence of the microspheres. HPLC traces obtained for these systems after irradiation confirmed that photodegradation of PCP takes place. The decrease in PCP concentration was found to be much faster in the presence of the microspheres which clearly indicate their photosensitizing activity. Moreover, the consumption of PCP is faster for higher microsphere concentration (Figure 3) and for microspheres with higher content of naphthyl chromophores (Figure 4). 


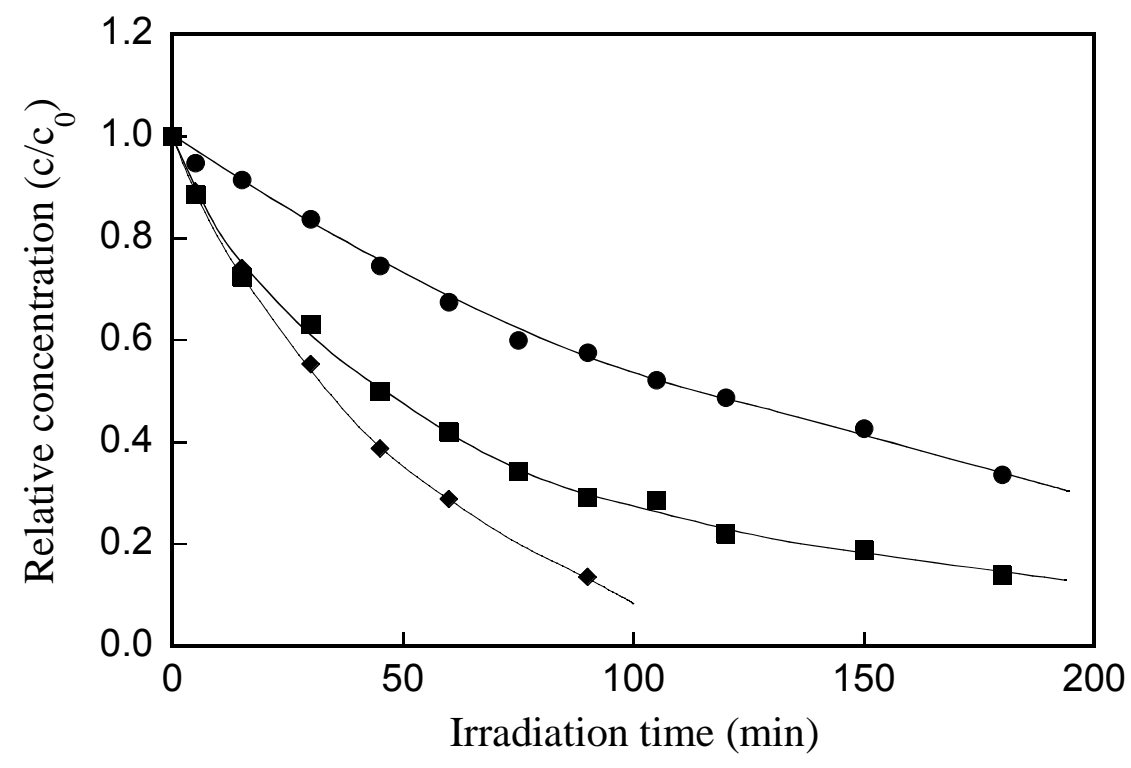

Fig. 3. Relative $\left(\mathrm{c} / \mathrm{c}_{0}\right)$ changes in PCP concentration in 15 vol.- $\%$ of methanol in water with irradiation time in the absence $(\bullet)$ and in the presence of NHNp1 microspheres at the concentration equal to $0,2(\boldsymbol{\square})$, and $4 \mathrm{~g} \cdot \mathrm{dm}^{-3}(\diamond)$.

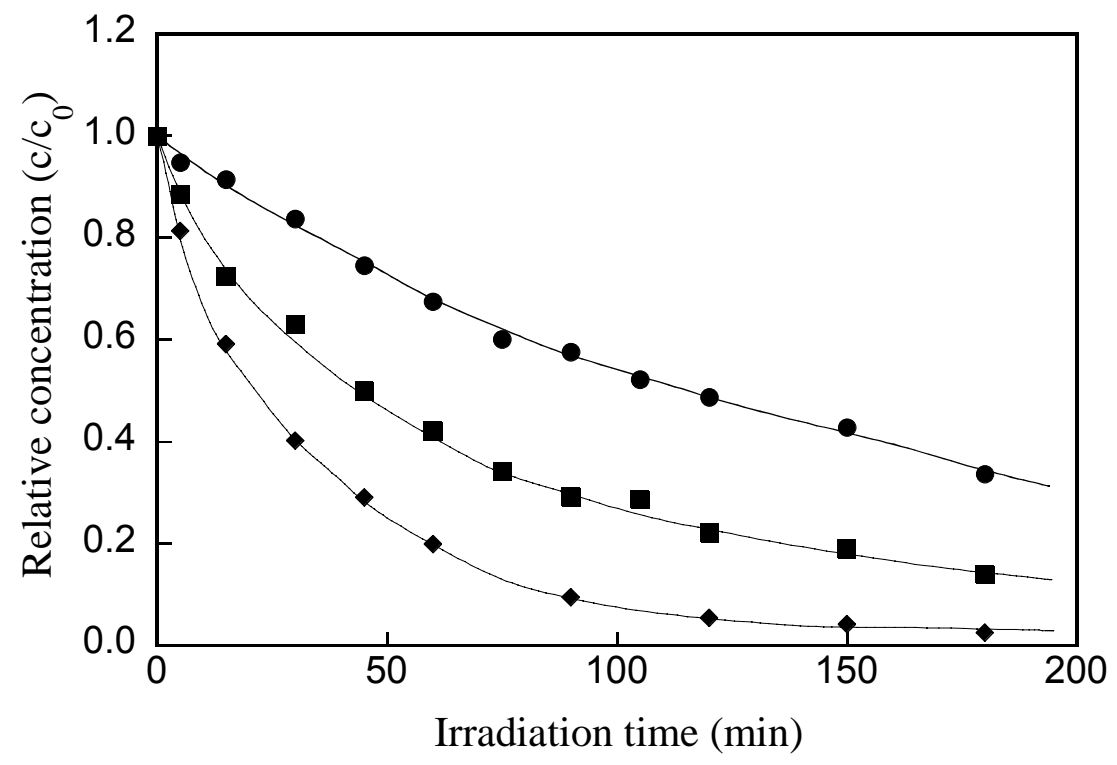

Fig. 4. The relative changes of PCP concentration in 15 vol.- $\%$ of methanol in water with irradiation time in the absence of the hydrogel $(-)$ and in the presence of $\mathrm{NHNp} 1(\square)$ and NHNp2 $(\bullet)$ microspheres at $\mathrm{c}_{\mathrm{p}}=2 \mathrm{~g} \cdot \mathrm{dm}^{-3}$.

The results demonstrated that PCP removal followed the pseudo-first order kinetics (Figure 5). The values of the rate constants for the reaction carried out in the absence and in the presence of 2 and $4 \mathrm{~g} \cdot \mathrm{dm}^{-3}$ of microspheres were found to be $6.4 \cdot 10^{-3}, 14.3 \cdot 10^{-3}$, and $21.5 \cdot 10^{-3} \mathrm{~min}^{-1}$, respectively. The HPLC analyses have shown that, the decrease in the intensity of PCP peak was accompanied by the appearance of new peaks at shorter retention times suggesting the presence of various reaction products (Figure 6). 


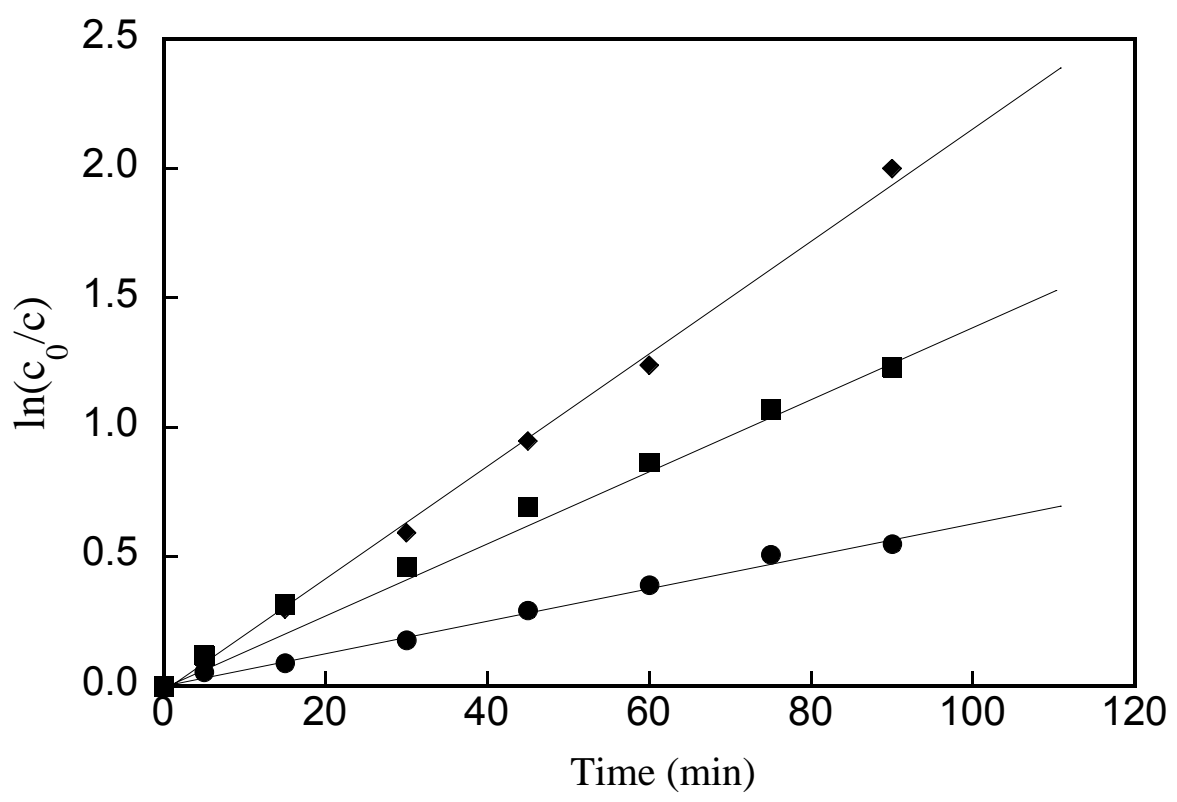

Fig. 5. Dependence of the PCP concentration on the irradiation time fitted to the first order kinetic equation in solutions containing $0(\mathbf{0}), 2(\boldsymbol{\square})$, and $4 \mathrm{~g} \cdot \mathrm{dm}^{-3}(\bullet)$ of NHNp1 microspheres.

Both in the presence and in the absence of the microspheres the products of the photoreaction are expected to be the lower chlorinated PCP isomers. This was concluded from the UV spectra of the products of the irradiated samples obtained with a PDA detector. The general shape of the absorption spectra of the products corresponded to those of chlorophenols, i.e. they display an intensive absorption band at $210-230 \mathrm{~nm}$ and a weaker band at $280-300 \mathrm{~nm}$. It was observed, that the long wavelength absorption band shifts to shorter wavelengths with decreasing retention time of the product peaks (data not shown). Such a shift in a position of absorption band is indeed observed for chlorinated phenols with decreasing chlorine content in a molecule. Another suggestion about the identity of the products is the fact that for the standards of chlorinated phenols available to us their peaks coeluted with the peaks of the products (e.g. 2,5-dichlorophenol, 2,4,6-trichlorophenol). The products of the photosensitized reaction have generally lower retention times possibly indicating a higher degree of dechlorination than the products of direct photodechlorination after the same irradiation time. That is important from the ecological point of view because lower chlorinated compounds are generally less toxic than those with higher chlorine content. It should be noted that the relative amounts of the products formed cannot be compared from the above chromatograms because they were collected using a UV absorption PDA detector at the absorption wavelength of $303 \mathrm{~nm}$ (the maximum of the long wavelength absorption band of PCP), while lower chlorinated phenols absorb at shorter wavelengths. No products were found at retention times longer than that of PCP in HPLC analyses which excludes the formation of the products with higher molecular weight (such as chlorinated dioxins, benzofurans, or biphenyls) being the usual products of condensation reactions, which are characteristic of the combustion of chlorinated phenols. 


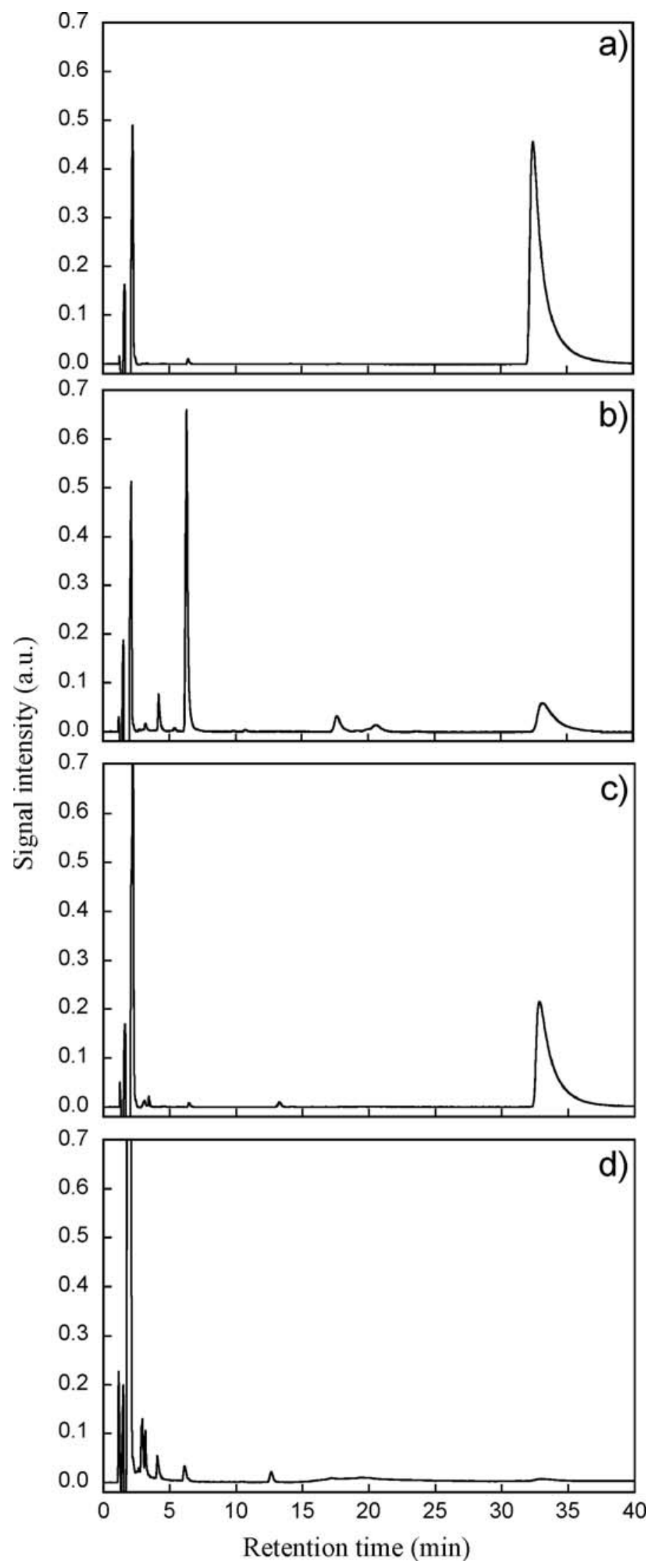

Fig. 6. HPLC chromatograms of the PCP solutions a) nonirradiated in the absence of the hydrogel, b) irradiated for $180 \mathrm{~min}$ in the absence of the hydrogel $\left(\lambda_{\text {abs }}=214.5\right.$ 
$\mathrm{nm}$ ), c) nonirradiated in the presence of NHNp2, and d) irradiated for 180 min in the presence of NHNp2 at $2 \mathrm{~g} \cdot \mathrm{dm}^{-3}$.

In order to determine the mechanism of photosensitized degradation of PCP by photoactive polymeric microspheres the interaction of PCP with the electronically excited naphthalene chromophores present in microspheres was studied using the fluorescence technique. It was found that PCP quenches naphthalene chromophores bound to polymer chain (Figure 7a). Singlet-singlet energy transfer or electron transfer between naphthalene chromophore (donor) and PCP (acceptor) can be considered as possible mechanisms for the observed phenomenon. The singletsinglet energy transfer from Np to PCP seems to have negligible contribution to the photosensitized dechlorination of PCP. This is because the energy of the excited singlet state of 1-methoxynaphthalene is $89.3 \mathrm{kcal} \cdot \mathrm{mol}^{-1}[31]$, while that for PCP in

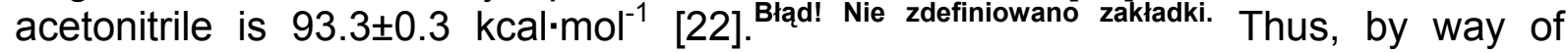
elimination, it can be concluded that quenching of microsphere-bound naphthalene chromophores by PCP is due to the electron transfer

$$
\mathrm{Np}^{*}+\mathrm{PCP} \rightarrow \mathrm{Np}^{+}+\mathrm{PCP}^{-}
$$

The free energy, $\Delta \mathrm{G}$, of the electron transfer process for this donor-acceptor pair can be calculated using the Rehm-Weller equation [32]:

$$
\Delta G=E_{D}{ }^{o x}-E_{A}^{r e d}-E^{*}-C
$$

where $E_{D}{ }^{o x}$ is the oxidation potential of the donor $\left(E_{D}{ }^{o x}=2.1 \mathrm{eV}\right)$ [33], $E_{A}{ }^{\text {red }}$ is the reduction potential of the acceptor $\left(E_{A}^{\text {red }}=-1.7 \mathrm{eV}\right)$ [34], $E^{*}$ is the energy of the excited state of the donor $\left(E^{*}=3.9 \mathrm{eV}\right)$ [33], and $\mathrm{C}$ is the electrostatic correction term, which is usually equal to $0.1 \mathrm{eV}$ in polar solvents [35]. The obtained value of $\Delta G$ is $-0.2 \mathrm{eV}$, which shows that the electron transfer is thermodynamically favorable. That first step (Eq.1), followed by the expelling of the chlorine anion, and is the most probable mechanism of the dechlorination of PCP:

$$
\mathrm{PCP}^{-} \rightarrow \mathrm{TCP}+\mathrm{Cl}^{-}
$$

where TCP is tetrachlorophenol.

The kinetics of the primary photochemical step can be determined by fitting of the experimental data of fluorescence quenching of NHNp2 microspheres by PCP to the Stern-Volmer equation (see Figure $7 \mathrm{~b}$ ). Based on that fit and assuming the value of lifetime of the singlet excited state of naphthyl chromophores attached to microspheres as equal to $13 \mathrm{~ns}$, which is a value determined for 1methoxynaphthalene (a model compound) [31], the rate constant of quenching process $\left(k_{\mathrm{q}}\right)$ was found to be $(5.5 \pm 0.5) \times 10^{11} \mathrm{dm}^{3} \cdot \mathrm{mol}^{-1} \cdot \mathrm{s}^{-1}$. That value is higher than expected for the diffusion controlled process in this solvent mixture (for water and methanol the rate of diffusion controlled energy transfer is $1.1 \times 10^{10}$ and 1.8 $\mathrm{x} 10^{10}$, respectively [31]). This suggests a significant contribution of static quenching of naphthalene chromophores by PCP.

It should be pointed out that the microspheres studied are thermosensitive, i.e. they show lower critical solution temperature (LCST) due to the content of NIPAM [30]. For example, we have found that they adsorb PCP from solution and that this adsorption is stronger above LCST of the polymer. Their thermal behavior, however, requires further study and will be presented in a separate paper. 

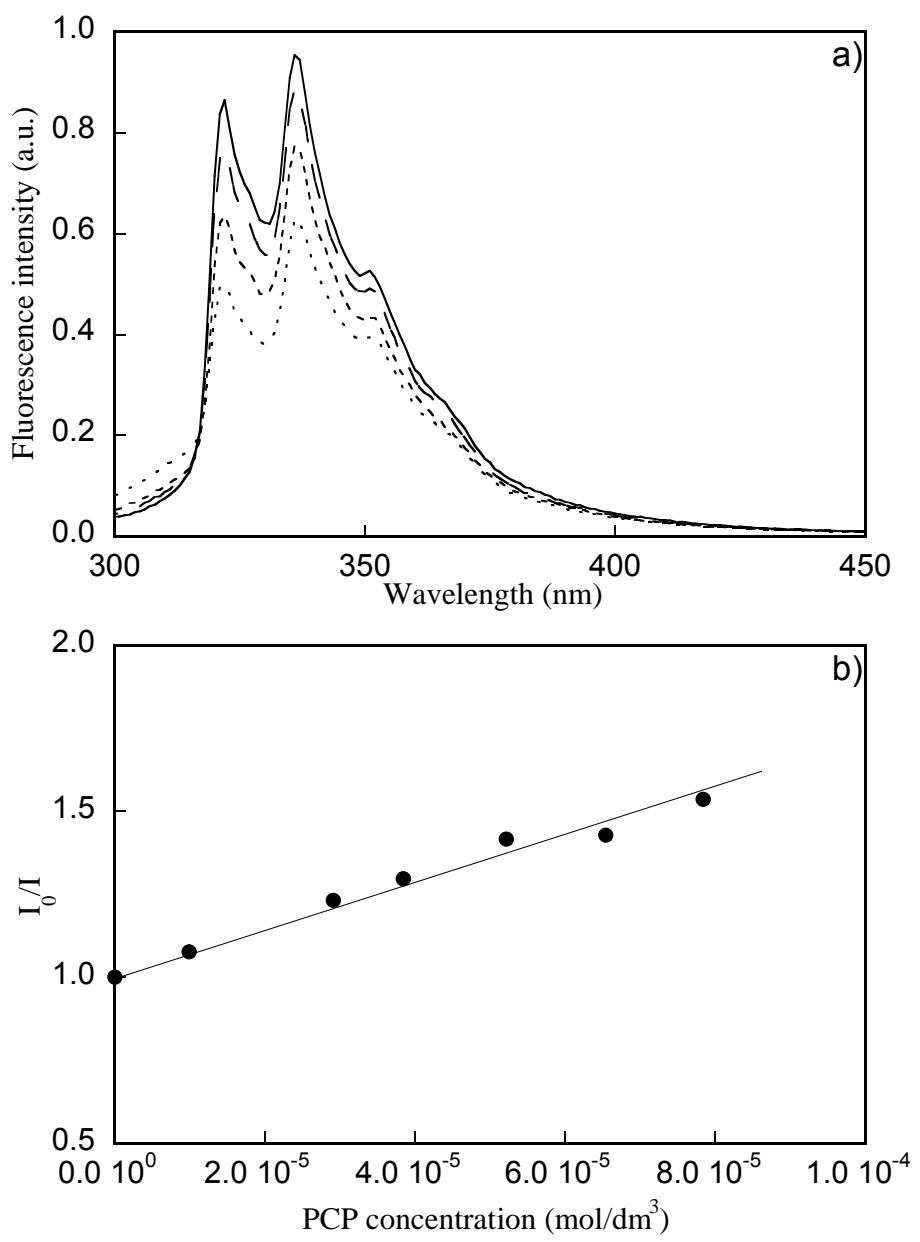

Fig. 7. a) The fluorescence emission spectra of $\mathrm{NHNp} 2$ in the absence (solid line) and in the presence of $9.90 \cdot 10^{-6}(--), 2.91 \cdot 10^{-5}(--\longrightarrow)$, and $9.09 \cdot 10^{-5}$ $\mathrm{mol} \cdot \mathrm{dm}^{-3}$ (- - - -) PCP b) The Stern-Volmer plot for the quenching of NHNp2 fluorescence by PCP $\left(c_{p}=0.1 \mathrm{~g} \cdot \mathrm{dm}^{-3}, \lambda_{\mathrm{ex}}=288 \mathrm{~nm}\right)$.

\section{Conclusions}

The hydrogel microspheres composed of a copolymer of NIPAM and HEMA were functionalized with naphthyl chromophores. The hydrogels were found to photosensitize the dechlorination of PCP, a model chlorinated compound, when irradiated with UV light in the aqueous solution. Lower chlorinated phenols were identified as products of both direct and photosensitized dechlorination of PCP. The molecular weights of the products of the photosensitized reaction were, however, lower compared to those of the dechlorination due to direct absorption after the same irradiation time. It is suggested that dechlorination takes place due to electron transfer from excited naphthyl chromophore to PCP followed by the release of the chlorine anion. Thus, the obtained hydrogel can be potentially used in the purification of water polluted with CAPs. 


\section{Experimental Part}

\section{Materials}

NIPAM ( $N$-isopropylacrylamide, Aldrich) was purified by dissolution in toluene followed by precipitation in a large excess of hexane and drying in vacuum, HEMA (2-hydroxyethyl methacrylate, Aldrich) was distilled under vacuum. Methylenebisacrylamide (BIS, Aldrich), N,N,N',N'-tetraethylmethylenediamine (TEMED, Fluka), 1-naphthyl glycidyl ether (NGE, Aldrich), ammonium persulfate (APS, POCH, Gliwice), sorbitan monopalmitate (Span 40, Sigma), sorbitan monooleate (Span 80, Sigma), 4-(N,N'-dimethylamine)pyridine (DMAP, Aldrich) and cyclohexane $(\mathrm{POCH}$, Gliwice) were used as received. Water was distilled twice. Pentachlorophenol (PCP, Aldrich) was recrystallized from methanol.

\section{Apparatus}

Ultraviolet-visible spectra were measured with a Hewlett-Packard 8452A diode array spectrophotometer. The fluorescence spectra were measured with an SLM-Aminco spectrofluorimeter. The elemental analysis $(\mathrm{C}, \mathrm{H}$, and $\mathrm{N})$ was performed with a Euroea 3000 elemental analyzer. HPLC analyses were performed using a Waters chromatograph equipped with a Symmetry C18 $5 \mu \mathrm{m}(4.6 \mathrm{~mm} \times 150 \mathrm{~mm})$ column and a Waters 2996 PDA detector. The eluent was 1:1 v/v mixture of water and acetonitrile containing 0.2 vol.- $\%$ of acetic acid. The flow rate was $1 \mathrm{~mL} \cdot \mathrm{min}^{-1}$. The photographs of the microspheres were taken using an optical microscope (Carl Zeiss Jena) equipped with a digital camera (Nikon Digital Net DN 100) and a Hitachi S4700 scanning electron microscope working at $10 \mathrm{kV}$. Fluorescence spectra of microspheres were measured using an SLM-AMINCO spectrofluorimeter using the excitation wavelength $\lambda_{\mathrm{ex}}=288 \mathrm{~nm}$. The SEM microphotographs were taken using the Hitachi S-4700 Scanning Electron Microscope.

\section{Synthesis of the Microspheres}

The microspheres were synthesized in a reverse suspension polymerization process based on the procedure described by $\mathrm{Ni}$ et al [30]. Cyclohexane $(65 \mathrm{ml})$ was poured into a $500 \mathrm{ml}$ three-necked flask equipped with a reflux and thermometer. Span 40 $(0.174 \mathrm{~g})$ and Span $80(0.087 \mathrm{~g})$ were added as stabilizers. Nitrogen was passed through the solution for 30 minutes. In a small beaker the solution of HEMA $(0.217 \mathrm{~g}$, $1.67 \mathrm{mmol}$ ), NIPAM (1.703 g, $15.04 \mathrm{mmol}$ ) (HEMA:NIPAM 1:9 mol/mol), and BIS ( $0.080 \mathrm{~g}, 0.52 \mathrm{mmol}, 3 \mathrm{~mol} \%$ based on total monomers) in $10 \mathrm{~mL}$ of water was prepared and degassed with nitrogen and poured into the flask. The solution of APS $(0.020 \mathrm{~g})$ and TEMED $(0.042 \mathrm{~g})$ in $1 \mathrm{~mL}$ of water was then added at the stirring speed of $1500 \mathrm{rpm}$. The temperature was increased slowly up to $70^{\circ} \mathrm{C}$ within $1 \mathrm{~h}$. The polymerization was continued overnight. The product was washed three times with acetone, three times with distilled water and freeze-dried. The HEMA:NIPAM molar ratio in the microspheres were close to that in the reaction mixture (10:90 vs. 9:91, respectively), as found from the elemental analysis.

\section{Functionalization of Microspheres}

Typical procedure was as follows: $1 \mathrm{~g}$ of the microspheres and $0.840 \mathrm{~g}$ of DMAP were added to $10 \mathrm{ml}$ of DMF. The suspension was heated to $50^{\circ} \mathrm{C}$ and $0.459 \mathrm{~g}$ of NGE dissolved in $5 \mathrm{ml}$ of DMF was added dropwise. Heating was continued for $12 \mathrm{~h}$. 
The microspheres were filtered out, washed in turn with acetone and water and dried in vacuum in $50^{\circ} \mathrm{C}$.

\section{Irradiations}

Irradiations were performed in a Rayonet RPR-100 photochemical reactor equipped with 4 or 8 lamps (RPR-3500, 24 W each) with the maximum emission intensity at $350 \mathrm{~nm}$. The samples were degassed by bubbling nitrogen for 30 minutes before the irradiation. During irradiation the sample was stirred with a magnetic stirrer.

\section{Acknowledgements}

The authors are grateful to the Polish State Committee for Scientific Research for the financial support in the form of Grant No. 7 T09A 13726.

\section{References}

[1] Encyclopedia of Smart Materials, Schwartz, M. Ed., John Wiley and Sons, Inc., New York, 2002.

[2] Luzinov, I.; Minko, S.; Tsukruk, V.V. Prog.Polym. Sci. 2004, 29, 635.

[3] Salvia, M. Ann.Chim-Sci.Mat. 2004, 29, 1.

[4] Tzou, H.S.; Lee, H.J.; Arnold, S.M. Mech.Adv.Mater.Struct. 2004, 11, 367.

[5] Roy, I.; Gupta, M.N. Chem.Biol. 2003, 10, 1161.

[6] Wojciechowski, S.; Boczkowska, A. Arch.Metall.Mater. 2004, 49, 723.

[7] Reguera, J.; Alonso, M.; Testera, A.M.; Lopez, I.M.; Martin, S.; RodriguezCabello, J.C. Carbohyd.Polym. 2004, 57, 293.

[8] Park, J.S.; Akiyama, Y.; Winnik, F.M.; Kataoka, K. Macromolecules 2004, 37, 6786.

[9] Flores-Ramirez, N.; Elizalde-Pena, E.A.; Vasquez-Garcia, S.R.; GonzalezHernandez, J.; Martinez-Ruvalcaba, A.; Sanchez, I.C.; Luna-Barcenas, G.; Gupta, R.B. J.Biomat.Sci.-Polym.Ed. 2005, 16, 473.

[10] El-Sayed, M.E.H.; Hoffman, A.S.; Stayton, P.S. J.Control.Rel. 2005, 101, 47.

[11] Dorfmann, A.; Ogden, R.W. Acta Mech. 2005, 174, 167.

[12] Sung, J.H.; Cho, M.S.; Choi, H.J.; Jhon, M.S. J.Ind.Eng.Chem. 2004, 10, 1217.

[13] Brigadnov, I.A.; Dorfmann, A. Continuum Mech.Therm. 2005, 17, 29.

[14] Shcherbakov, V.P.; Winklhofer, M. Phys.Rev. E 2004, 70, art.no. 061803.

[15] Toensmeier, P.A. Plast.Eng. 2005, 61, 10.

[16] El-Sayed, M.E.H.; Hoffman, A.S.; Stayton, P.S. Expert Opin.Biol.Ther. 2005, 5, 23.

[17] Piskin, E.; Dincer, S.; Turk, M. J.Biomater.Sci.-Polym.Ed. 2004, 15, 1181.

[18] Piskin, E. Int.J.Pharm. 2004, 277, 105.

[19] Johnson, B.D.; Beebe, D.J.; Crone, W. Mater.Sci.Eng.C-Biomimetic.Supramol. Syst. 2004, 24, 575.

[20] Hoffman, A.S.; Stayton, P.S. Macromol.Symp. 2004, 207, 139.

[21] Nowakowska, M.; Kępczyński, M.; Szczubiałka, K. Macromol.Chem.Phys. 1995, 196, 2073.

[22] Nowakowska, M.; Szczubiałka, K. J.Photochem.Photobiol.A 1995, 91, 81.

[23] Nowakowska, M.; Szczubiałka, K.; Zapotoczny, Sz. J.Photochem.Photobiol.A 1996, 97, 93.

[24] Nowakowska, M.; Szczubiałka, K. Chemosphere 1999, 39, 71. 
[25] Nowakowska, M.; Sterzel, M.; Zapotoczny, S.; Kot, E. Appl.Catal.B: Environ. 2005, 57, 1.

[26] Nowakowska, M.;.Zapotoczny, S.; Sterzel, M.; Kot, E. Biomacromolecules, 2004, 5, 1009.

[27] Nowakowska, M.; Sterzel, M.; Szczubiałka, K. J.Polym.Environ. 2006, 146, 59.

[28] Nowakowska, M.; Karewicz, A.; Kłos, M.; Zapotoczny, S. Macromolecules, 2003, $36,4134$.

[29] Nowakowska, M.; Kępczyński, M.; Szczubiałka, K. Pure Appl.Chem. 2001, 73, 491.

[30] Ni, C.; Wang, Z.; Zhu, X. J.Appl.Polym.Sci. 2004, 91, 1792.

[31] Murov, S. Handbook of Photochemistry, Marcel Dekker, New York, 1973.

[32] Weller, A. Z.Phys.Chem., Neue Folge 1982, 133, 93.

[33] Eberson, L.; Radner, F. J.Am.Chem.Soc. 1991, 113, 5825.

[34] Ross, N.C.; Spackman, R.A.; Hitchman, M.L.; White, P.C. J.Appl.Electrochem. 1997, 27, 51.

[35] Rehm, D.; Weller, A. Z.Phys.Chem., Neue Folge 1970, 69, 183. 